\title{
False susceptibility of antibitoics to carbapenemase producers and means to overcome
}

\author{
Manu Chaudhary and Anurag Payasi* \\ Department of Cell Culture and Molecular Biology, Venus Medicine Research Centre, Baddi, H.P. - 173205 \\ India
}

\begin{abstract}
The aim of this study was to identify the reason for frequent failure of carbapenems and $\beta$-lactam and $\beta$-lactamase inhibitor combination drugs in clinical settings despite susceptibility. For this different isolates collected from clinical specimens were tested for susceptibility and identification of carbapenemase producers was done by phenotypic method (Modified Hodge Test). Further, phenotypic results were compared with the Gold standard PCR results to assess accuracy of the results.

A total of 541 isolates collected from various centres across India including Escherichia coli $(n=154)$, Klebsiella pneumoniae $(n=161)$, Acinetobacter baumannii $(n=103)$ and Pseudomonas aeruginosa $(n=123)$ were included in the study. These isolates were tested for suceptibility according to Clinical and Laboratory Standards Institute (CLSI) methods and then screened for carbapenemase following the same guidelines. The isolates were then subjected to Modified Hodge Test with and without zinc sulfate with subsequent confirmation with the genotypic assay.

Out of 541 clinical isolates, 464 isolates were identified as probabale carbapenemase producers. Further phenotypic screening of these isolates, only 316 (68.1\%) isolates were found to be positive to MHT. After addition of zinc into MHT sensitivity of the test was improved and now 431 (92.9\%) isolates were found to be positive to MHT indicating it is $24.8 \%$ more effective compared to MHT without zinc sulfate. Subsequently, when 316 MHT positive isolates were screened through PCR, $30 \%$ (95/316) isolates showed false positive and $19.6 \%$ (29/316) isolates showed false negative, results indicating higher degree of false positive and negative results are associated with MHT. However, with the addition of zinc sulfate to MHT, false positive and negative results are minimized significantly. Among the tested drugs, Elores appeared to be the most efficacious with 90.9 $\%$ to $95.4 \%$ susceptibility to carbapenemase producing organisms. Intrestingly, penems (meropenem and imipenem plus cilastatin) exhibited higher resistance varing from $90 \%$ to $96.1 \%$. For the proper treatment of patients, it is important to use simple and reliable tests for identification of carbapenemase producing clinical isolates among gram negative organisms. This study showed that the MHT technique is highly sensitive for detecting carbapenemases after addition of zinc. When susceptibility of various drugs were tested against carbapenemase producing isolates, Elores was found as the most efficacious drugs.
\end{abstract}

Keywords: NDM-1, Carbapenemases, Modified Hodge Test.

\section{Introduction}

Carbapenems, $\beta$-lactam group of antimicrobials, are being used worldwide as the last resort antibacterial agents for the treatment of infections caused by multi-drug resistant (MDR) gram negative organisms. In comparison to earlier $\beta$-lactam antibacterial agents, carbapenems have broader spectrum activity and are stable to hydrolysis against $\beta$-lactamases [1]. However, in recent years, resistance to carbapenems among gram-negative pathogens has been emerging steadily and thus putting clinical application of this antibiotic under threat [2]. Resistance to carbapenem is mainly mediated by carbapenemases such as Ambler class B metallo- $\beta$-lactamases (MBL), including IMP, VIM, and NDM-1 which have been increasingly reported in Enterobacteriaceae and Pseudomonas spp. [3-5]. Carbapenemase enzymes are usually encoded by mobile DNA elements with a high capacity for dissemination [6]. Infections due to carbapenemases producing strains are associated with higher morbidity and mortality rates [7].

As carbapenem resistance is a growing problem worldwide, therefore its identification is of importance for the determination of appropriate therapeutic regimens and implementation of infection control measures [8]. Carbapenemase gene detection by polymerase chain reaction is gold standard but needs skilled technician and is available in only in a few laboratories, thereby limiting its use. In addition to that, several phenotypic techniques have been employed for detection of carbapenemases production [8-9] but in vitro tests for detection of carbapenemase activity do not possess high sensitivity and specificity and may show false positive results, particulalry, when multiple mechanisms of resistance occur in bacterial strains as well as when isolates exhibit low-level of carbapenem resistance [10]. Therefore, a quick, reliable and affordable laboratory method for the detection of carbapenemase-producing enterobacteriaceae is of utmost importance in any clinical laboratory.

Taking into account the high rate of clinical failures among penems and other $\beta$-lactam and $\beta$-lactam 
inhibitor (BL and BLI) combination drugs, we tried to modify this MHT technique for improving its detection limits. As MBLs are zinc dependent (Walsh et al., 2005), zinc sulfate was added to Mueller-Hinton agar (MHA) at concentration of $70 \mu \mathrm{g} / \mathrm{ml}$ in order to increase sensitivity of the test. The aim of this study was to identify the carbapenemase producers among isolates collected from different clinical specimens by phenotypic method (Modified Hodge Test). Further, phenotypic results were compared with the Gold standard PCR results to assess the accuracy of the results. Subsequently, susceptibility study was carried out in these isolates with commonly used $\beta$-lactam drugs.

\subsection{Bacterial strains}

\section{Materials And Methods}

A total of 541 isolates including Escherichia coli $(\mathrm{n}=154)$, Klebsiella pneumoniae $(\mathrm{n}=161)$, Acinetobacter baumannii $(\mathrm{n}=103)$ and Pseudomonas aeruginosa $(\mathrm{n}=123)$ recovered from various clinical specimens were included in the study. These isolates were recovered from various parts of India including Uttar Pradesh, Delhi, Kolkata, Haryana, Hyderabad and Mumbai. Identity of the isolates was confirmed with VITEK 2. These isolates were incoculated in Mueller-Hinton broth for overnight at $37^{\circ} \mathrm{C}$ prior to use. These strains were further tested for carbapenemase production.

\subsection{Antibiotic susceptibility testing}

Carbapenemase producing isolates are considered to be multi-drug resistant, hence, we further tested the antimicrobial susceptibility of these isolates for various antibiotics including ceftriaxone plus sulbactam; Elores $(30: 15 \mu \mathrm{g})$, piperacillin plus tazobactam $(100: 10 \mu \mathrm{g})$, imipenem plus cilastatin $(10 \mu \mathrm{g})$, and meropenem $(10 \mu \mathrm{g})$ by the disc diffusion method as recommended by Clinical and Laboratory Standards Institute (CLSI, 2013). All the discs were obtained from Hi-Media Laboratories Pvt. Ltd., Mumbai, India.

\subsection{Screening for carbapenemases production}

All the isolates were exposed to identify carbapenemases production according to the methods developed by CLSI guidelines (2013). The isolates showing zone of inhibition of $\leq 23 \mathrm{~mm}$ with imipenem or meropenem were selected for the phenotypic method by modified Hodge test (MHT) and genotypic method by polymerase chain reaction (PCR).

\subsection{Phenotypic detection of carbapenemase production by modified Hodge test (MHT)}

MHT test was performed according to the CLSI guidelines [11]. A 1:10 dilution of $0.5 \mathrm{Mc}$ - Farland suspension of E. coli ATCC 25922 was streaked as lawn on to a Müller-Hinton agar plate. A $10 \mu \mathrm{g}$ meropenem or imipenem susceptibility disk was applied in the center of the lawn. The test organism and positive/negative control were streaked starting from the edge of the carbapenem disc towards the edge of the plate. The plate was incubated overnight at $35 \pm 2^{\circ} \mathrm{C}$ for $16-24$ hours. The plates were examined after $16-24$ hours of incubation for a clover leaf type indentation at the intersection of the test organism and control stain E.coli 25922 within the zone of inhibtion of the carbapenem susceptibility disk. MHT positive test with a clover-leaf indentation of the E. coli 25922 growing along the test organism growth streak within the disk diffusion zone indicated that the isolate was producing a carbapenemase. MHT negative test with no growth of the E. coli 25922 along the test organism growth streak and no growth within the disc diffusion indicated that the isolate was not producing a carbapenemase. MHT is a simple test for screening for MBL production but occasional false negative results have been reported. In order to increase the sensitivity of test zinc sulpahte at the concentration of $70 \mu \mathrm{g} / \mathrm{ml}$ was added to th MH agar plates for MHT. K. pneumoniae ATCC BAA 1705 and K. pneumoniae ATCC BAA 1706 were used as positive and negative controls, respectively.

\subsection{DNA isolation}

DNA of all chosen isolates was extracted following the method described previously [12]. Five $\mathrm{ml}$ of over night grown culture of each isolate was used for the DNA isolation. DNA purity and concentrations were measured with spectrophotometer (260/280).

\subsection{Molecular detection of the MBL genes}

The isolated DNA of all clinical isolates were exposed to PCR to detect NDM-1, VIM-1 and IMP-1. The detection of these genes was carried out by using the primers reported previously [13]. PCR amplification was performed in a total volume of $20 \mu \mathrm{l}$ containing $200 \mathrm{pg}$ of DNA, $0.5 \mathrm{mM}$ of dNTPs, $1.25 \mu \mathrm{M}$ of each primer and $1.5 \mathrm{U}$ of Taq polymerase (Banglore Genei). PCR amplification was done using Eppendorf thermocycler (Germany). The amplicon was analyzed on $1 \%(\mathrm{w} / \mathrm{v})$ agarose gel supplemented with ethidium bromide. 


\subsection{Identification of clinical isolates}

\section{Results}

The isolates were confirmed to be E. coli $(\mathrm{n}=154), K$. pneumoniae $(\mathrm{n}=161)$, A. baumannii $(\mathrm{n}=103)$ and $P$. aeruginosa $(\mathrm{n}=123)$. Out of a total of 541, 464 were found to have inhibition zone diameter of less than 23 $\mathrm{mm}$ and subjected for further characterization. PCR results confirmed that 431 including E. coli $(\mathrm{n}=120), K$. pneumoniae $(\mathrm{n}=133)$, A. baumannii $(\mathrm{n}=80)$ and P. aeruginosa $(\mathrm{n}=98)$ to be of carbapenemase producers which includes NDM-1, VIM-1 and IMP-1 (Figure 1).

\subsection{Phenotypic detection of carbapenemase by Modified Hodge test}

Phenotypic detection for carbapenemase production was further done by Modified Hodge test without and with incorporation of zinc sulphate (Figure 2 and 3). Of 464 isolates, MHT was found to be positive in 316 $(68.1 \%)$ isolates and negative in $148(31.9 \%)$ isolates in the absence of zinc sulphate. After addition of zinc into MHT sensitivity of the test was improved and we noted respectively $431(92.9 \%)$ and $33(7.1 \%)$ isolates were positive and negative to MHT. Through PCR, carbapenemase genes were detected in $431(92.9 \%)$ isolates. As PCR is considered as gold standard, when the isolates which were positive for MHT in the absence of zinc sulphate were re-evaluated with PCR, only $47.6 \%$ (221/464) isolates were found to be positive by PCR (true positive) and therefore positive predictive value would be $69.9 \%(221 / 316)$, while $30 \%(95 / 316)$ isolates were found to be negative to PCR (false positive) therefore false positive predictive value would be $30 \%$.

In contrast, $25.6 \%$ (119/464) isolates in the absence of zinc sulphate were found to be negative by PCR (true negative) and therefore negative predictive value would be $80.4 \%$ (119/148) whereas $19.6 \%$ (29/148) isolates were PCR positive (false negative) therefore false negative predictive value would be $19.6 \%$ (29/148). These results indicate that false positive $(30 \%)$ and false negative $(19.6 \%)$ results are associated with Hodge test. However, it was observed that with the addition of zinc sulphate positive predictive value accuracy increases from $69.9 \%$ to $100 \%$ whereas negative predictive valuse accuracy ranges from $80.4 \%$ to $100 \%$. This shows with the addition of zinc sulphate, sensitivity of Hogde test is increased significantly.

\subsection{Prevalence of different carbapenemases among clinical isolates}

Our results showed that out of $80 \mathrm{~A}$. baumannii isolates, $15(18.7 \%)$ isolates were confirmed to be positive for NDM-1; 11 (13.7\%) isolates were IMP-1 positive; 18 (22.5\%) were positive for VIM-1; $22(27.5 \%)$ were co-producers of both NDM-1 and VIM-1; 14 (17.5\%) were NDM-1+IMP-1+VIM-1. Among 120 isolates of $E$. coli, NDM-1 was detected in $45(37.5 \%)$ isolates; $12(10 \%)$ isolates had IMP-1; VIM-1 was evident in 20 $(16.6 \%)$ isolates; $27(22.5 \%)$ isolates were found to carry NDM-1+IMP-1; NDM-1+IMP-1+VIM-1 was detected in $16(13.3 \%)$ isolates. Of 133 isolates of $K$. pneumoniae, NDM-1 was evident in $37(27.8 \%)$ isolates; $16(12 \%)$ isolates horboured IMP-1; $33(24.8 \%)$ isolates were positive to VIM-1; NDM-1+VIM-1 was evident in $25(18.8 \%)$ isolates; NDM-1+IMP-1+VIM-1 was identified in $22(16.5 \%)$ isolates. Out of 98 isolates of $P$. aeruginosa, NDM-1 was present in 26 (26.5\%) isolates; 13 (13.2\%) isolates had IMP-1; VIM-1 was prevalent in $19(19.4 \%)$ isolates; $14(14.3 \%)$ isolates were found to carry NDM-1+IMP-1 and NDM-1+VIM-1 was evident in $26(26.5 \%)$ isolates (Table 1$)$.

\subsection{Antibiotic susceptibility testing}

Results of antimicrobial susceptibility are shown in Table 2. Susceptibility results revealed that among the tested drugs, Elores was found to be more efficacious. Approximately, $91.6 \%$ to $95 \%$ of the E. coli isolates harboring various carbapenemases were susceptible to Elores when tested by disc diffusion method followed by K. pneumoniae (91 to 94\%), P. aerugionsa (92.3 to $94.7 \%$ ) and A. baumannii (90.9 to $95.4 \%$ ). None of the isolates was susceptible to meropenem, imipenem plus cilastatin and piperacillin plus tazobactam. Piperacillin+tazobactam showed 87.5 to $96.3 \%$ resistance against carbapenemase producing isolates whereas exhibited 3.7 to $12.5 \%$ intermediate response. Surprisingly, our results showed a marked changes in susceptibility patterns on penems (meropenem and imipenem) which exhibited 90 to $96.1 \%$ resistant and 4.4 to $11.1 \%$ intermediate response to carbapenemase producing isolates.

\section{Discussion}

Over the past years, increasing incidence of carbapenemase producing Enterobacteriaceae, Pseudomonas and Acinetobacter has been reported globally [14-18]. Of the carbapenemases, NDM-1, IMP-1, and VIM-1 type carbapenemase has been reported recently in many countries [19-20]. In this investigation we found that more than $85 \%$ isolates were carbapenem resistant of which $68.1 \%$ of isolates were phenotypically MBL positive which corroborates with previous study where Hodge test identified only $67 \%$ cases of MBL producing $P$. aeruginosa and Acinetobacter spp [21]. To our surprize $30 \%$ false positive and $19.6 \%$ false negative results are associated with MHT test for MBL detection. These $30 \%$ false positive results lead to false susceptibility of penems and failue of clinical treatment with either of these drugs may complicate the case and 
result in mortality or worsening of patient condition. Similarly, $19.6 \%$ false negative exclude the use of penems on these patients which could have otherwise been treated well.

All the patients from whom these isolates were recovered succumbed to serious infections. After confirmation of carbapenemase, they were treated with appropriate antibiotics and get recovered soon. Microbiologists and clinicians should be aware of the false results when only MHT are used to screen isolates. However, as reported earlier [9], we observed that these false results could be overcome by adding zinc into MHT. Earlier, Poirel et al., [22] from Argentina have reported false susceptibility in class A carbapenemases using MHT which was enhanced using boronic acid compound. Our results showed that MHT with zinc could identify 115 other isolates, which were not detected by MHT without zinc. The results obtained with MHT in the presence of zinc sulfate appeared to be accurate and reliable for the diagnosis of carbapenemases and the evaluation of treatment response in most carbapenemase producing bacterial infection. Thus, it had higher sensitivity with more positive predictive value for carbapenemase detection. Although Rasheed et al., [23] reported a low MHT sensitivity for NDM-1 producers (50\%) which increased to $85.7 \%$ by adding $\mathrm{ZnSO}_{4}$ (100 $\mu \mathrm{g} / \mathrm{ml}$ ) in the culture medium, still PCR is the only $100 \%$ confirmatory test which is not possible to perform due to paucity of time and chornicity of patient. The high positive predictive value of MHT with zinc in our study suggest to be used this method as an effective tool for screening of carbapenemase in enterobacteriaceae. This is not only cost-effective but also can be utilized for an early detection using bacterial isolates recovered directly from the clinical specimens. Therefore, there is a need for alternative fast detection methods for MBLs to assist clinicains in choosing appropriate therapy right the first time.

Our data also demonstrated, there were increasing trend of carbapenemases in clinical isolates. Our previous studies also noted the steadily increasing frequency of carbapenemases in E. coli, K. pneumoniae, A. baumannii and $P$. aeruginosa [24-27]. An earlier study from India also reported high penem resistance [28]. An earlier study from Australia in A. baumannii clinical isolates reported $64 \%$ resistance to meropenem [29], where as approximately $75 \%$ of NDM-1producing $K$. pneumoniae and all were resistant to ertapenem, imipenem, and meropenem [30]. A still higher 100\% resistance to imipenem was reported in P. aeruginosa [31] .

\section{Conclusion}

It is important to use simple and reliable tests for identification of carbapenemase producing clinical isolates among gram negative organisms for proper selection of antibiotic therapy. Organisms producing carbapenemase remain an important cause for therapy failure with commonly used drugs due to false susceptibility patterns. This study showed that the MHT technique is highly sensitive for detecting class carbapenemases after addition of zinc. Study also highlighted an alrming rise in MBLs in Indian population where penems and BL+ BLI combinations fail to perform and, Elores was found as the most efficacious drug.

\section{Acknowledgement}

Authors are thankful to sponsor, Venus Medicine Research Centre, Werne, Germany, for providing financial assistance to carry out this study, and also thankful to institute which provided clinical isolates.

\section{References}

[1] A. Amjad, I.A. Mirza, S.A. Abbasi, U. Farwa, N. Malik, and F. Zia, Modified Hodge test: a simple and effective test for detection of carbapenemase production. Iranian Journal of Microbiology, ,2011,189- 193.

[2] M. Chaudhary, and A. Payasi, Prospective study for antimicrobial susceptibility of Escherichia coli isolated from various clinical specimens in India. Journal of Microbial and Biochemical Technology, 4, 2012, 157-160.

[3] M. Chaudhary, and A. Payasi, Antimicrobial susceptibility patterns and molecular characterization of Klebsiella pneumoniae clinical isolates from north indian patients. International Journal of Medicine and Medical Sciences, 46, 2013, 1218-1224.

[4] M. Chaudhary, and A. Payasi, Incidence, prevalence and control of multidrug resistant (MDR) carbapenemase producing Acinetobacter baumanii in Indian intensive care units. Journal of Pharmacy Research, 7, 2013, 175-180.

[5] M. Chaudhary, and A. Payasi, Rising antimicrobial resistance of Pseudomonas aeruginosa isolated from clinical specimens in India. Journal of Proteomics and Bioinformatics, 6, 2013, 005-009.

[6] M. Chaudhary, and A. Payasi, Elores : a new antibiotic adjuvant entity active against metallo- $\beta$-lactamases producing organisms. International Journal of Medicine and Medical Sciences, 46, 2013, 1145-1150.

[7] Clinical and Laboratory Standards Institute. Performance standards for antimicrobial susceptibility testing; twenty-third informational supplement. CLSI document M100-S23. 2013, Wayne, PA 19087 USA

[8] M.N. Kim, S.H. Park, D. Ahn, and M. Lee, Emergence and epidemiology of New Delhi metallo-lactamase1-producing Klebsiella pneumoniae in a Korean tertiary care hospital. Abstract number: P647, Abstracts of 21st ECCMID / 27th IC,Milan, Italy, 7-10 May 2011. European Society of Clinical Microbiology and Infectious Diseases.

[9] D. Gilrich, L. Poirel, and P. Nordmann, Value of the Modified Hodge test for detection of emerging carbapenemases in Enterobacteriaceae. Journal of Clinical and Microbiology, 50, 2012, 477-479.

[10] P. Khuntayaporn, P. Montakantikul, P. Santanir, P. Kiratisin, and M.T. Chomnawant, Molecular investigation on carbapenems resistance among multidrug-resistant Pseudomonas aeruginosa clinical isolated in Thailand," Microbiology Immunology ,doi: $10.1111 / 1348-0421.12021,2012$.

[11] K.K. Kumarasamy, M.A. Toleman, T.R. Walsh, J. Bagaria, F. Butt, R. Balakrishanan, U. Chaudhary, M. Doumith, C.G. Giske, S. Irfan, P. Krishnan, A.V. Kumar, S. Maharjan, S. Mushtaq, T. Noorie, D.L. Paterson, A. Pearson, C. Perry, R. Pike, B. Rao, U. Ray, J.B. Sarma, M. Sharma, E. Sheridan, M.A. Thirunarayan, J. Turton, S. Upadhayay, M. Warner, W. Welfare, D.M. Livermore, and 
N. Woodford, Emergence of a new antibiotic resistance mechanism in India, Pakistan, and the UK: a molecular, biological, and epidemiological study. The Lancet Infectious Diseases, 10, 2010, 597-602.

[12] K. Lee, Y.S. Lim, D. Yong, J.H. Yum, and Y. Chong, Evaluation of the Hodge test and the imipenem-EDTA double-disk synergy test for differentiating metallo-beta-lactamase-producing isolates of Pseudomonas spp. and Acinetobacter spp. Journal of Clinical Microbiology, 41, 2003, 4623-4629.

[13] P. Majewski, P. Sacha, P. Wieczorek, D. Ojdana, A. Michalska, and E. Tryniszewska, New Delhi Metallo- $\beta$-Lactamases-the dawn of a post-antibiotic era. Progress in Health Science, 2, 2012, 153-160.

[14] H. Mammeri, H. Guillon, E.B. François, and P. Nordmann, Phenotypic and biochemical comparison of the carbapenemhydrolyzing activities of five plasmid-borne AmpC beta-lactamases. Antimicrobial Agents and Chemotherapy, 54, 2010, 45564560 .

[15] D.K. Mendiratta, V. Deotale, and P. Narang, Metallo- $\beta$-lactamase producing Pseudomonas aeruginosa in a hospital from a rural area. Indian Journal Medical Research, 121, 2005, 701-703.

[16] N. Mesaros, P. Nordmann, P. Ple'siat, M. Roussel-Delvallez, J. Van Eldere, Y. Glupczynski, Y. Van, Laethem, F. Jacobs, P. Lebecque, A. Malfroot, P.M. Tulkens, and F. Van Bambeke, Pseudomonas aeruginosa: resistance and therapeutic options at the turn of the new millennium. Clinical Microbiology and Infection, 13, 2007, $560-578$.

[17] V. Miriagou, G. Cornaglia, M. Edelstein, I. Galani, C.G. Giske, M. Gniadkowski, E. Malamou-Lada, F. Navarro, P. Nordmann, S. Pournaras, G.M. Rossolini, A. Tsakris, A. Vatopoulos, and R. Canton, Acquired carbapenemases in Gram-negative bacterial pathogens: detection and surveillance issues. Clinical Microbiology and Infection, 16, 2010, 112-122.

[18] P. Nordmann, L. Dortet, and L. Poirel, 2012. Carbapenem resistance in Enterobacteriaceae: here is the storm! Trends Mol. Med. 18, 2012, 263-272.

[19] A.Y. Peleg, C. Franklin, J.M. Bell, and D.W. Spelman, Emergence of carbapenem resistance in Acinetobacter baumannii recovered from blood cultures in Australia. Infection Control and Hosp ital Epidemiology, 27, 2006, 759-61.

[20] F. Pasteran, T. Mendez, M. Rapoport, L. Guerriero, and A. Corso, Controlling false-positive results obtained with the Hodge and Masuda assays for detection of class A carbapenemase in species of Enterobacteriaceae by incorporating boronic acid. Journal of Clinical Microbiology, 48, 2010, 1323-1332.

[21] S.S. Andrade, A.G. Nicoletti, E.H. Campana, G.C. Moraes, R.E. Mendes, and A.C. Gales, Metallo--lactamase detection: comparative evaluation of double-disk synergy versus combined disk tests for IMP-, GIM-, SIM-, SPM-, or VIM-producing isolates. J. Clin. Microbiol. 46, 2008, 2028-2037.

[22] L. Poirel, T.R. Walshb, V. Cuvillier, and P. Nordmann, Multiplex PCR for detection of acquired carbapenemase genes. Diagnostic Microbiology and Infectious Disease, 70, 2011, 119-123.

[23] J.K. Rasheed, B. Kitchel, W. Zhu, K.F. Anderson, N.C. Clark, M.J. Ferraro, P. Savard, R.M. Humphries, A.J. Kallen, and B.M. Limbago, New Delhi Metallo- $\beta$-Lactamase-producing Enterobacteriaceae, United States. Emerging Infectious Diseases, 19, 2013, 870-878.

[24] J.M.Rolain, P. Parola, and G..Cornaglia, "New Delhi metallo-b-lactamase (NDM-1): towards a new pandemi," Clinical Microbiology and Infections, 16, 2010, $1699-1701$.

[25] B. Suh, I. K. Bae, J. Kim, S. H. Jeong, D. Yong, and K. Lee. Outbreak of meropenem-resistant Serratia marcescens comediated by chromosomal AmpC beta-lactamase overproduction and outer membrane protein loss. Antimicrob. Agents Chemother., 54, 2010, 5057-61.

[26] R. Tellis, S. Muralidharan, and A.I. Peter, Evaluation of phenotypic methods fro the detection of metallo-beta-lactamases production in non-fermenting gram-negative bacilli. International Journal of Biomedical and Advance Research, 4, 2013, 299304.

[27] J. Sambrook, and D.W. Russell, Molecular cloning: a laboratory manual. New York: Cold Spring Harbor Laboratory Press 2001.

[28] M. Souli, I. Galanil, A. Antoniadou, E. Papadomichelakis, Poulakou G, Panageal T, Vouril S, Zerva L, armaganidis A, Kanellakopoulou K, and Giamarellou H, An outbreak of infection due to beta-lactamase Klebsiella pneumoniae carbapenemase 2-producing K. pneumoniae in a Greek university hospital: molecular characterization, epidemiology, and outcomes. Clinical Infectious Disease, 50, 2010, $364-373$.

[29] B.A. Sultan, E. Khan, F. Hussain, A. Nasir, and S. Irfan, Effectiveness of Modified Hodge Test to detect NDM-1 Carbapenemases: an experience from Pakistan. Journal of Pakistan Medical Association, 63, 2013;955-960.

[30] T.R. Walsh, M.A. Toleman, L. Poirel, and P. Nordmann, Metallo beta lactamases: the quiet before the storm? Clinical Microbiology Review, 18, 2005, 306-325.

[31] D. Yong, M.A. Toleman, C.G. Giske, H.S. Cho, K. Sundman, K. Lee, and T.R. Walsh, "Characterization of a new metallo -betalactamase gene, bla(NDM-1), and a novel erythromycin esterase gene carried on a unique genetic structure in Klebsiella pneumoniae sequence type 14 from India," Antimicrobial Agents and Chemotherapy, 53, 2009, 5046 -54.

Table 1: Prevalence of various carbapenemase among different clinical isolates.

\begin{tabular}{|l|c|c|c|c|}
\hline \multirow{2}{*}{ Carbapenemase genes } & \multicolumn{4}{|c|}{ Carbapenemase \% } \\
\cline { 2 - 5 } & $\begin{array}{c}\text { A. baumannii } \\
(80)\end{array}$ & E. coli $(120)$ & K. pneumoniae $(133)$ & $\begin{array}{c}\text { P. aeruginosa } \\
(98)\end{array}$ \\
\hline NDM-1 & $18.7(15)$ & $37.5(45)$ & $27.8(37)$ & $26.5(26)$ \\
\hline IMP-1 & $13.7(11)$ & $10(12)$ & $12(16)$ & $13.2(13)$ \\
\hline VIM-1 & $22.5(18)$ & $16.6(20)$ & $24.8(33)$ & $19.4(19)$ \\
\hline NDM-1+IMP-1 & - & $22.5(27)$ & - & $14.3(14)$ \\
\hline NDM-1+VIM-1 & $27.5(22)$ & - & $18.8(25)$ & $26.5(26)$ \\
\hline NDM-1+IMP-1+VIM-1 & $17.5(14)$ & $13.3(16)$ & $16.5(22)$ & - \\
\hline
\end{tabular}


Figure 1:Agarose gel showing PCR amplified products of carbapenemase genes.
$\mathbf{A}$
B
C
D
$\mathbf{E}$
$\mathbf{F}$
G

Lane A 100 bp DNA size marker; Lane B to C NDM-1(621 bp); lane D to E IMP-1 (190 bp); lane F to G VIM-1 (390 bp).

Figure 2: Summary of the screening of isolates.

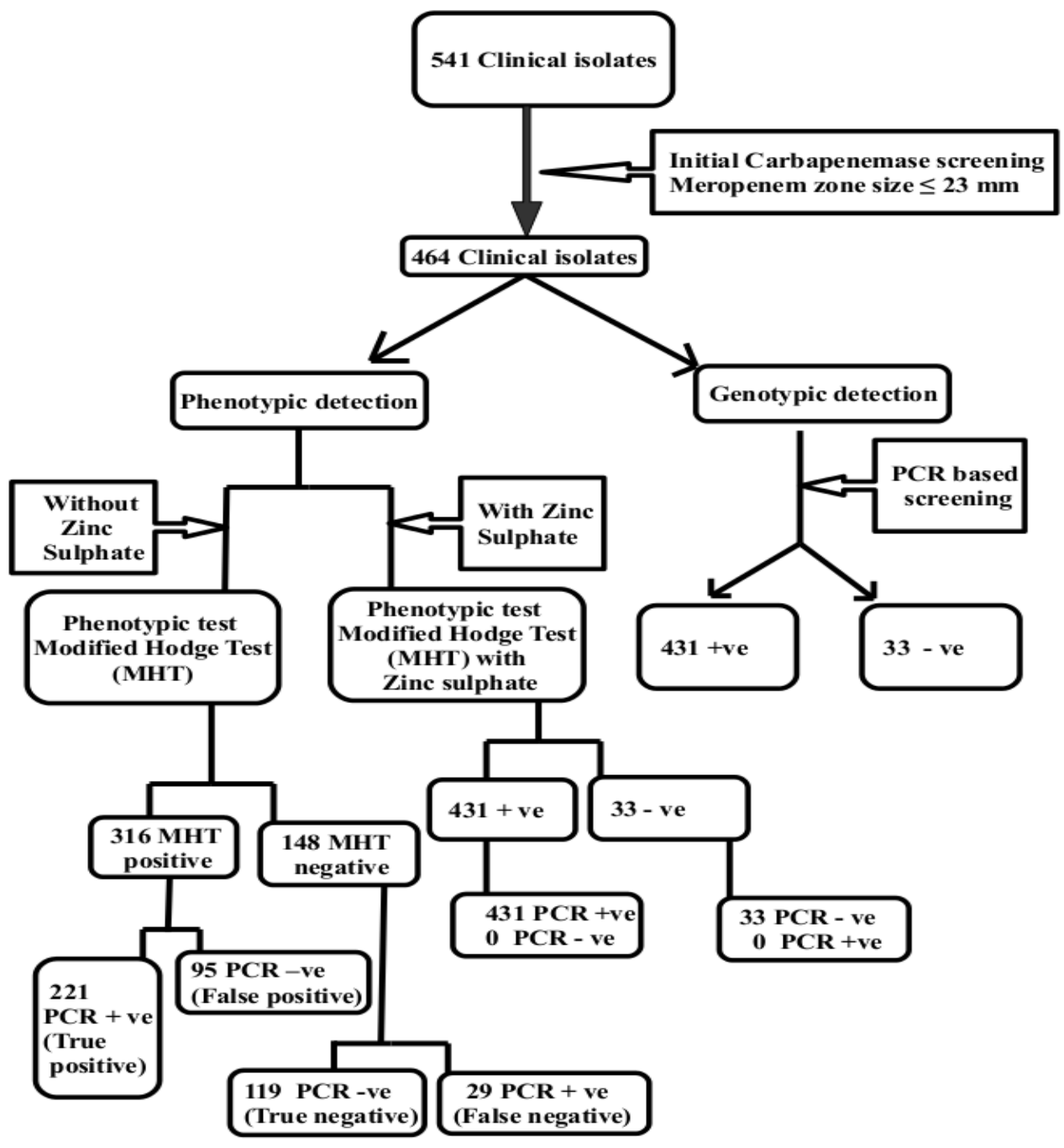

MHT test: Positive predictive value : $69.9 \%$; Negative predictive value : $82.4 \%$

MHT with zinc sulphatetest: Positive predictive value : 100\%; Negative predictive value : 100\% 
Figure 3: The MHT performed on a Muller Hinton agar plate. (1) MHT negative result (2) MHT positive result (positive control (3) and (4) clinical isolate (positive result).

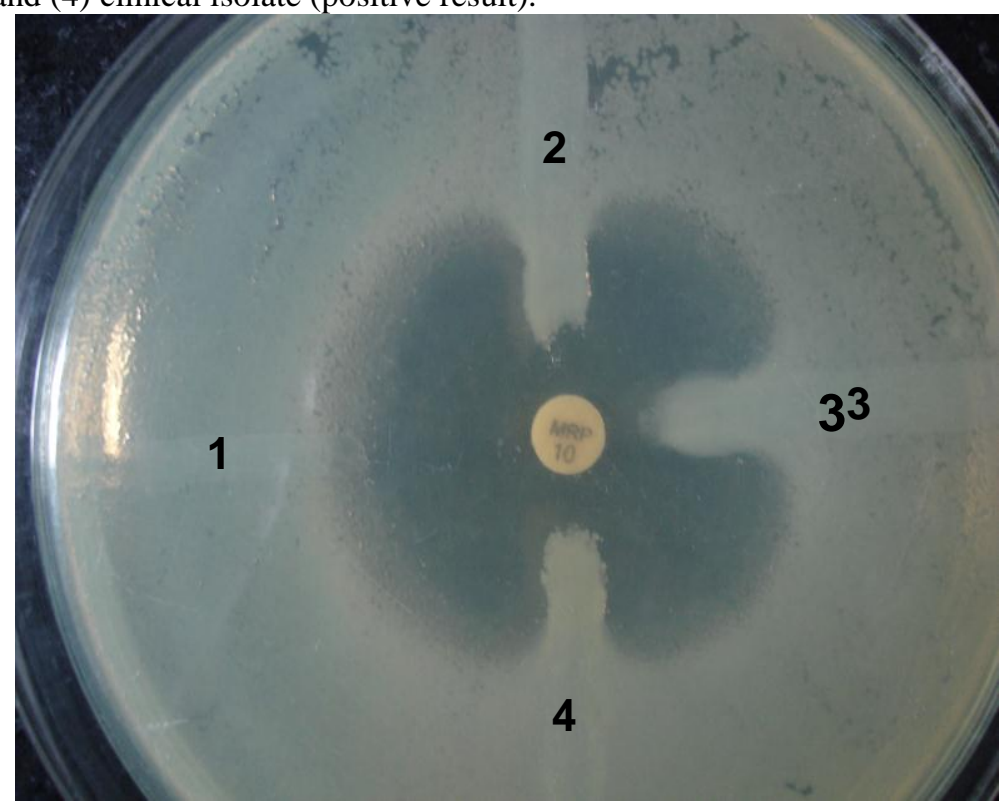

Table 2: Percentage of antibiotic susceptibility of carbapenemase positive clinical isolates.

\begin{tabular}{|c|c|c|c|c|c|c|c|c|c|c|c|c|c|}
\hline & & \multicolumn{3}{|c|}{ A. baumannii } & \multicolumn{3}{|c|}{ E. coli } & \multicolumn{3}{|c|}{ K. pneumoniae } & \multicolumn{3}{|c|}{ P. aeruginosa } \\
\hline & & $S$ & I & $\mathrm{R}$ & S & I & $\mathrm{R}$ & $S$ & I & $\mathrm{R}$ & S & I & $\mathrm{R}$ \\
\hline \multirow[t]{6}{*}{ Elores } & NDM-1 & 93.3 & - & 6.6 & 93.3 & 2.2 & 4.4 & 91.9 & 2.7 & 5.4 & 92.3 & 3.8 & 3.8 \\
\hline & IMP-1 & 90.9 & - & 9.1 & 91.6 & - & 8.3 & 93.7 & - & 6.2 & 92.3 & - & 7.7 \\
\hline & VIM-1 & 94.4 & - & 5.5 & 95 & - & 5 & 94 & 3 & 3 & 94.7 & 5.2 & - \\
\hline & NDM-1+IMP-1 & 94.4 & - & 5.5 & 92.6 & 3.7 & 3.7 & 91 & 3 & 6 & 92.8 & - & 7.1 \\
\hline & NDM-1+VIM-1 & 95.4 & - & 4.5 & 95 & - & 5 & 92 & 4 & 4 & 92.3 & 3.8 & 3.8 \\
\hline & NDM-1+IMP-1+VIM-1 & 92.8 & - & 7.1 & 93.7 & - & 6.2 & 91 & 4.5 & 4.5 & 94.7 & - & 5.2 \\
\hline \multirow[t]{6}{*}{ Meropenem } & NDM-1 & - & 6.6 & 93.3 & - & 6.6 & 93.3 & - & 8.1 & 91.9 & - & 7.7 & 92.3 \\
\hline & IMP-1 & - & 9.1 & 90.9 & - & 8.3 & 91.6 & - & 6.2 & 93.7 & - & 7.7 & 92.3 \\
\hline & VIM-1 & - & 11.1 & 88.8 & - & 5 & 95 & - & 94 & 6 & - & 5.3 & 94.7 \\
\hline & NDM-1+IMP-1 & - & 5.5 & 94.5 & - & 7.4 & 92.6 & - & 9.1 & 90.9 & - & 7.1 & 92.8 \\
\hline & NDM-1+VIM-1 & - & 4.5 & 95.4 & - & 10 & 90 & - & 8 & 92 & - & 7.7 & 92.3 \\
\hline & NDM-1+IMP-1+VIM-1 & - & 7.1 & 92.8 & - & 6.2 & 93.7 & - & 9.1 & 90.9 & - & 5.2 & 94.7 \\
\hline \multirow{6}{*}{$\begin{array}{l}\text { Imipenem+cilas } \\
\text { tatin }\end{array}$} & NDM-1 & - & 6.6 & 93.3 & - & 4.4 & 95.5 & - & 5.4 & 94.6 & - & 3.8 & 96.1 \\
\hline & IMP-1 & - & 9.1 & 90.9 & - & 8.3 & 91.6 & - & 6.2 & 93.7 & - & 7.7 & 92.3 \\
\hline & VIM-1 & - & 5.5 & 94.4 & - & 5 & 95 & - & 6 & 94 & - & 5.2 & 94.7 \\
\hline & NDM-1+IMP-1 & - & 5.5 & 94.5 & - & 7.4 & 92.6 & - & 6 & 94 & - & 7.1 & 92.8 \\
\hline & NDM-1+VIM-1 & - & 4.5 & 95.4 & - & 5 & 95 & - & 8 & 92 & - & 7.7 & 92.3 \\
\hline & NDM-1+IMP-1+VIM-1 & - & 7.1 & 92.8 & - & 6.2 & 93.7 & - & 9.1 & 90.9 & - & 5.2 & 94.7 \\
\hline \multirow{6}{*}{$\begin{array}{l}\text { Piperacillin+ } \\
\text { tazobactam }\end{array}$} & NDM-1 & - & 6.6 & 93.3 & - & 4.5 & 95.5 & - & 8.1 & 91.9 & - & 7.7 & 92.3 \\
\hline & IMP-1 & - & 9.1 & 90.9 & - & 8.3 & 91.6 & - & 12.5 & 87.5 & - & 7.7 & 92.3 \\
\hline & VIM-1 & - & 5.5 & 94.4 & - & 5 & 95 & - & 6 & 94 & - & 5.2 & 94.7 \\
\hline & NDM-1+IMP-1 & - & 5.5 & 94.4 & - & 3.7 & 96.3 & - & 9.1 & 90.9 & - & 7.1 & 92.8 \\
\hline & NDM-1+VIM-1 & - & 4.5 & 95.4 & - & 10 & 90 & - & 8 & 92 & - & 7.7 & 92.3 \\
\hline & NDM-1+IMP-1+VIM-1 & - & 7.1 & 92.8 & - & 6.2 & 93.7 & - & 4.5 & 95.4 & - & 5.2 & 94.7 \\
\hline
\end{tabular}

